\title{
Low surface brightness galaxies around the HDF-S ${ }^{\star}$
}

\section{Distances and volume densities}

\author{
L. Haberzettl ${ }^{1,2}$, D. J. Bomans ${ }^{1}$, and R.-J. Dettmar ${ }^{1}$ \\ 1 Astronomical Institute Ruhr-University Bochum, Universitätsstrasse 150, 44780 Bochum, Germany \\ 2 Present address: Department of Physics and Astronomy, University of Louisville, Louisville, KY 40292, USA \\ e-mail: lghabe01@louisville.edu
}

Received 11 December 2006 / Accepted 24 May 2007

\section{ABSTRACT}

\begin{abstract}
Aims. With this study we aim at the spectroscopic verification of a photometrically selected sample of Low Surface Brightness (LSB) galaxy candidates in a field around the Hubble Deep Field-South (HDF-S). The sample helps to extend the parameter space for LSB galaxies to lower central surface brightnesses and to provide better estimates on the volume densities of these objects.

Methods. To derive redshifts for the LSB candidates, long-slit spectra were obtained covering a spectral range from $3400 \AA$ to $7500 \AA$. The observations have been obtained using the ESO $3.6 \mathrm{~m}$ telescope, equipped with the EFOSC2 spectrograph. From the measured radial velocities, distances could be estimated. With this distance information, it is possible to differentiate between true LSB galaxies and higher redshift High Surface Brightness (HSB) galaxies which may contaminate the sample. A correction for the surface brightnesses can then be applied, accounting for the cosmological dimming effect ("Tolman Dimming").

Results. We show that $\sim 70 \%$ of the LSB candidates, selected based on their location in the color-color space, are real LSB galaxies. Their position in the color-color diagrams, therefore, indicate that the LSB galaxies have a different stellar population mix resulting from a different star formation history compared to HSBs. Our LSB galaxy sample consists only of large disk galaxies with scalelength between $2.5 \mathrm{kpc}$ and $7.3 \mathrm{kpc}$. We confirm the flat central surface brightness distribution of previous surveys and extend this distribution down to central surface brightnesses of $27 \mathrm{~B}$ mag $\operatorname{arcsec}^{-2}$.
\end{abstract}

Key words. surveys - galaxies: distances and redshifts - galaxies: fundamental parameters

\section{Introduction}

The known properties of LSB galaxies are still a challenge for existing theories of galaxy formation and evolution. Although they may not represent a significant amount of the luminosity density in the universe, their number density is very high. Searches for LSB galaxies showed that they account for up to $60 \%$ of the local galaxy population (McGaugh 1996; O'Neil \& Bothun 2000). Minchin et al. (2004) showed in their study of the bivariate surface brightness distribution, that up to $20 \%$ of the dynamical mass is represented by the LSB galaxies and that they account for up to $60 \%$ of the number density in the Universe. Thus it seems, that LSB galaxies are a common product of galaxy formation and evolutionary processes. Therefore, it is essential to understand their role in formation and evolution scenarios of galaxies in general.

Studies of the properties of local LSB galaxy samples have shown that they populate nearly the whole parameter space derived also for HSB galaxies. The only difference is their low central surface brightness which is below $\mu_{0}=22.5 \mathrm{mag} \mathrm{arcsec}^{-2}$ in the $B$-filter. Field LSB galaxies are generally gas rich, although the gas surface densities are very low, too. The typical gas surface densities for LSB galaxies are below the Kennicutt criterion for ongoing star formation (Kennicutt 1989; van der Hulst et al. 1993; Pickering et al. 1997) which results in a suppressed current star formation rate. The morphology of LSB galaxies does not show any significant differences to the morphology of

$\star$ Full Fig. 1 is only available in electronic form at http://www . aanda. org
HSB galaxies. The properties of LSBs are independent of the Hubble type, these galaxies exist over the whole Hubble sequence (Schombert et al. 1992). While the LSB galaxies could be found in all morphological types and results are comparable to results from surveys governed by HSB galaxies, e.g. UGC catalog (Nilson 1973), the major part of the population consists of late-type galaxies. Elliptical LSB galaxies are rare in these surveys $(\sim 14 \%)$ and found mostly in cluster environment, which means they underwent a different star formation history than field LSB galaxies.

The simplest evolutionary scenario suggests that LSB galaxies are faded remnants of HSB galaxies. For this scenario one would expect that LSB galaxies are found to have red colors. This scenario could be ruled out by the fact that LSB galaxies are found to cover the whole color space from very red to very blue. Most of the LSB galaxies have quite blue colors which could be explained only partly as the result of the relatively low metalicities $\left(Z<1 / 3 Z_{\odot}\right.$, e.g. Roennback \& Bergvall 1995; McGaugh 1993). De Blok et al. (1995) showed that their sample of 20 LSB galaxies tend to have bluer colors compared to the mean values estimated for HSB galaxies of the corresponding Hubble type. This effect is mainly seen in the $B-R$ color (HSB: $\overline{B-R}=0.92$, LSB: $\overline{B-R}=0.78$ ) and the $V-I$ color (HSB: $\overline{V-I}=0.90$, LSB: $\overline{V-I}=0.76$ ). These blue colors are a first hint for relatively young luminosity weighted average ages of the stellar populations in the LSB galaxies. However, until now we do have only little information about the evolutionary paths taken by the LSB galaxies, resulting in such low surface 
brightnesses (e.g. Gerritsen \& de Blok 1999; Bell et al. 2000; van den Hoek et al. 2000).

In this paper we present spectroscopic results for the LSB candidate sample derived in a field around the HDF-S (Haberzettl et al. 2007). We used spectroscopic measurements of redshifted emission lines to derive distances for these objects. From the distances we calculate physical parameters of the derived galaxies and distinguish the real LSB galaxies from the higher redshifted background objects. We also discuss the behavior of the LSB galaxies in the color-color space. Finally we compare our findings to results of previous searches for LSB galaxies.

\section{Observation and data reduction}

\subsection{Observations}

We performed spectroscopic observations of a sample of 9 LSB galaxy candidates during the nights of October 23rd-25th 2000 at the ESO $3.6 \mathrm{~m}$ telescope on La Silla. The spectra were obtained using the ESO Faint Object Spectrograph 2 (EFOSC2) equipped with a Loral $2048^{2}$ pixel $^{2}$ $\mathrm{CCD}$ and a pixel size of $15 \mu \mathrm{m}(0.157 \mathrm{arcsec})$. We took long-slit spectra using a grism of $300 \mathrm{gr} \mathrm{m}^{-1}$ (grism \# 11), resulting in a spectral sampling of $2.12 \AA \mathrm{pixel}^{-1}$. With this setup we were able to cover a spectral range from $3400 \AA$ to $7500 \AA$. The CCD was binned by a factor of 2 in both directions. This setup resulted in a spectral resolution of $\sim 4 \AA \mathrm{pixel}^{-1}$. The spectrograph was equipped with a long-slit of slightly less than 5 arcmin length in the spatial direction. For the observation we chose a slit width of 1.2 arcsec. This led to a FWHM for emission lines of $\sim 15.6 \AA$ at $4000 \AA$ in the derived spectra.

In order to minimize flux losses due to atmospheric differential refraction (Filippenko 1982), the observations were carried out in an airmass range between 1.1 and 1.8. This resulted in a refraction below 1.8 arcsec at $3500 \AA$ and above -0.9 arcsec at $7500 \AA$. Due to the sizes of the observed objects $(\sim 10$ to $\sim 30$ arcsec) these are still acceptable values. During the nights of the 23rd and 24th, the observing conditions were not photometric with a mean seeing of $\sim 2$ arcsec. In the last night, the conditions were nearly photometric with a seeing below 1 arcsec. Bias frames, dome flats, twilight-sky flats, and He-Ne comparison lamp exposures were taken at the beginning and the end of each night. For flux calibration, spectroscopic standard stars (Hamuy et al. 1992) were observed several times during each night. For the observations of the standard stars, we used the maximum slit width of 5 arcsec in order to increase the signal to noise ratio of the spectra as much as possible. Each science exposure was obtained with an exposure time of about $1800 \mathrm{~s}$. In most cases, two exposures per object were observed (see Table 1).

\subsection{Reduction and calibration}

The data reduction and calibration were carried out based on the standard reduction procedures within IRAF (Massey 1997; Massey et al. 1992). The wavelength calibration resulted in a spectral sampling of $4.04 \AA$ per pixel with an rms for the wavelength fit between $0.3 \AA$ and $1.0 \AA$ per pixel. After the wavelength calibration, we combined the single science exposures of every object. This coaddition makes it also possible to remove cosmic rays from the science spectra. The cosmic ray cleaning of LSB J22353-60311, for which we only observed one spectra, was done using the cosmic ray identification task L.A.
Table 1. Exposure times and number of exposures for the HDF-S LSB candidate sample observed with ESO $3.6 \mathrm{~m}$ telescope.

\begin{tabular}{ccc}
\hline \hline Name & Exposures time [s] & Exposures \\
\hline LSB J22311-60503 & 1800 & 2 \\
LSB J22324-60520 & 1800 & 2 \\
LSB J22325-60155 & 1800 & 2 \\
LSB J22330-60543 & 1800 & 2 \\
LSB J22343-60222 & 1800 & 2 \\
LSB J22352-60420 & 1800 & 2 \\
LSB J22353-60311 & 1800 & 1 \\
LSB J22353-60122 & 1800 & 2 \\
LSB J22355-60183 & 1800 & 2 \\
\hline
\end{tabular}

cosmic (van Dokkum 2001). This cosmic ray cleaning is based on a Laplace filtering method. In a next step we flux calibrated the coadded and cosmic ray cleaned spectra using the observed standard star spectra. Finally, we applied an extinction correction to the science spectra. We used only the foreground extinction value of $E(B-V)=0.028$ for this correction, which we adopted from the extinction maps of Schlegel et al. (1998). Applying only foreground extinction from the galaxy gives reasonable results for the LSB galaxies, since internal extinction can be neglected for these galaxies due to the low dust content. There exists only a few week detections for LSB galaxies in the FIR and mm-wavelength region where dust radiates thermal emission (Hoeppe et al. 1994). The final result of the reduction and calibration processes are 2D- and 1D-spectra which will be used in the following analysis.

\section{Results and discussion}

\subsection{Distances and physical parameters of the LSB sample}

Most of the common internal parameters of the studied galaxies e.g., sizes in physical units (radii, scale-length), luminosities, absolute magnitudes (see Table 3) are distance-dependent parameters. The radii are measured by eye, following the light distribution into the noise. This means that the radius is measured at the limiting surface brightness of $\sim 29 \mathrm{mag} \mathrm{arcsec}^{-2}$ (see Haberzettl et al. 2007). We chose the radii at the limiting surface brightness, because the Holmberg radius at $25 \mathrm{mag} \operatorname{arcsec}^{-2}$ would give no useful information about the sizes of LSB galaxies due to the low surface brightnesses in these galaxies. In order to derive the distances, we estimated the redshifts $z$ for our sample galaxies as the mean of the redshifts calculated for every well detected emission line in the single galaxy spectra.

In order to maximize the signal-to-noise ratios we summed up all columns along the spatial axis which contributed a significant signal to the final science spectra. In these integrated 1D spectra (see Fig. 1), it was now possible to detect the most important emission lines e.g., [OII], $\mathrm{H} \beta$, [OIII]-doublet, and $\mathrm{H} \alpha$. In some cases, we also detected the [S II]-doublet and derived useful upper limits for the [N II] 66584 emission line.

For the determination of the mean redshift, we used only emission lines which were clearly detected and which were not influenced by night sky emission lines. The lines which are only measured as upper limits or which were influenced by night sky emission lines are marked with ${ }^{a}$ in Table 2. Due to the low resolution, it was not possible to resolve the [SII] $\lambda \lambda 6717,6731$-doublet. Therefore, we also did not use this doublet for the estimation of the mean redshift and marked them with ${ }^{a}$ in Table 2 . The errors of the mean redshifts were calculated using the standard deviation. 

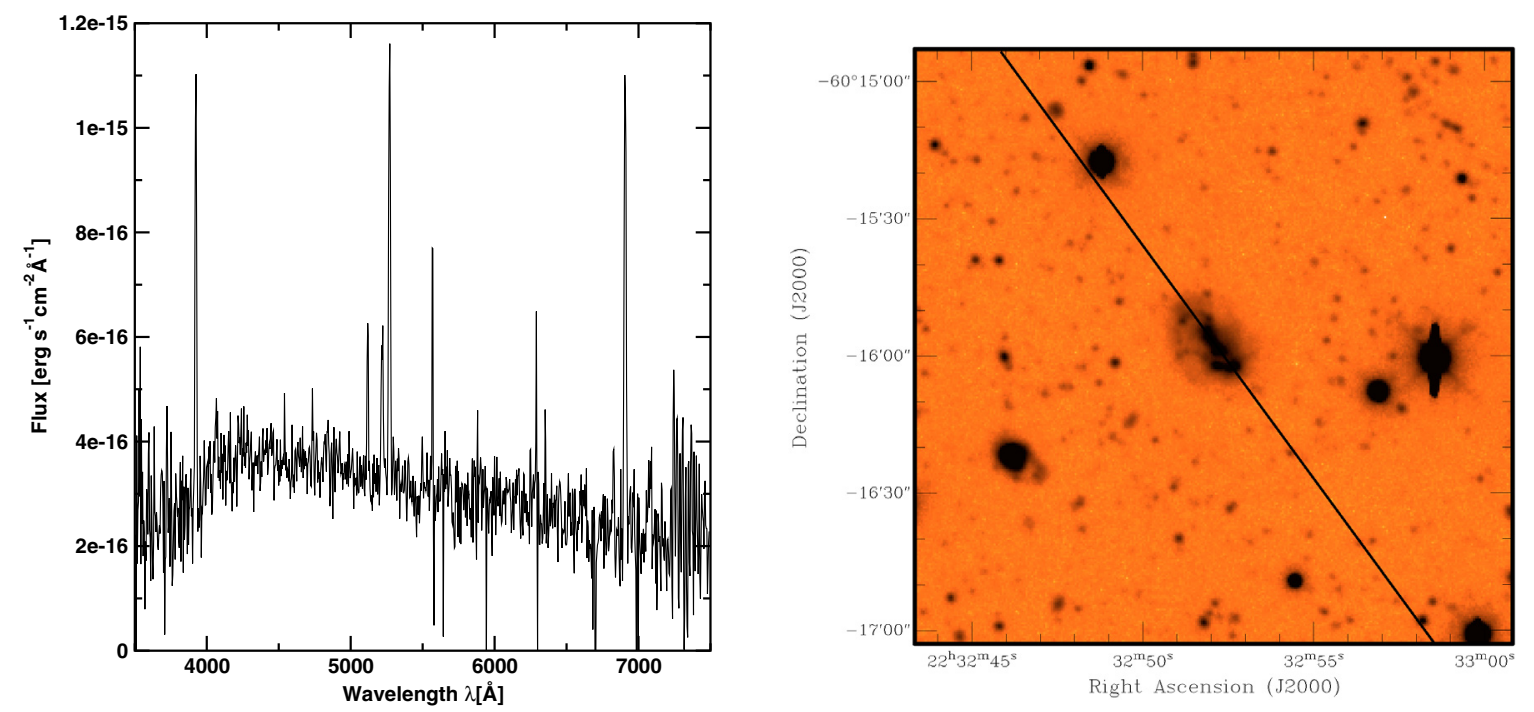

Fig. 1. In the following we show the 1D spectra and images of the spectroscopically observed LSB galaxy candidates in the HDF-S (e.g. LSB J22325-60155). The orientation and location of the slit is stated by the black line in the $2^{\prime} \times 2^{\prime}$ large images.

Table 2. Measurements for the emission lines of the LSB candidate sample. All entries which are marked with ${ }^{a}$ were not used for the estimation of the mean redshift $\langle z\rangle$ and therefore also not for the estimation of the standard deviation $\sigma_{z}$. All lines which were either influenced by night sky emission lines or which had only upper limits were also not used for the determination of the mean redshift. It was not possible to resolve the two component of the [SII]-doublet. Therefore [SII] was not used in order to estimate redshifts. The redshift of LSB J22324-60520 (marked with ${ }^{b}$ ) was estimated measuring the wavelength of the CaII-K absorption line.

\begin{tabular}{|c|c|c|c|c|c|c|c|c|c|}
\hline NAME & $\begin{array}{l}{[\mathrm{OII}]} \\
\lambda 3727 \\
{[\AA]}\end{array}$ & $\begin{array}{l}\mathrm{H} \beta \\
\lambda 4861 \\
{[\AA]}\end{array}$ & $\begin{array}{l}\text { [OIII] } \\
\lambda 4959 \\
{[\AA]}\end{array}$ & $\begin{array}{l}{[\mathrm{OIII}]} \\
\lambda 5007 \\
{[\AA]}\end{array}$ & $\begin{array}{l}\mathrm{H} \alpha \\
\lambda 6563 \\
{[\AA]}\end{array}$ & $\begin{array}{l}{[\mathrm{NII}]} \\
\lambda 6584 \\
{[\AA]}\end{array}$ & $\begin{array}{l}{[\mathrm{SII}]} \\
\lambda \lambda 6717,6731 \\
{[\AA]}\end{array}$ & $z$ & $\sigma_{z}$ \\
\hline LSB J22311-60503 & 4172 & - & - & & 7351 & - & - & 0.1198 & 0.0004 \\
\hline LSB J22325-60155 & 3924 & 5118 & 5219 & 5270 & 6907 & - & $7077^{a}$ & 0.0526 & 0.0002 \\
\hline LSB J22330-60543 & 3936 & 5133 & 5236 & 5287 & 6929 & - & $7096^{a}$ & 0.0559 & 0.0001 \\
\hline LSB J22343-60222 & 3903 & 5089 & 5190 & 5242 & 6866 & $6888^{a}$ & $7037^{a}$ & 0.0467 & 0.0005 \\
\hline LSB J22352-60420 & 4170 & 5439 & $5550^{a}$ & $5603^{a}$ & 7347 & - & - & 0.1191 & 0.0003 \\
\hline LSB J22353-60311 & 3946 & 5146 & 5250 & 5297 & 6943 & $6968^{a}$ & - & 0.0584 & 0.0004 \\
\hline LSB J22354-60122 & 4329 & 5647 & 5758 & 5816 & - & - & - & 0.1615 & 0.0002 \\
\hline LSB J22355-60183 & 4194 & 5468 & $5584^{a}$ & 5632 & 7381 & - & - & 0.1249 & 0.0003 \\
\hline LSB J22324-60520 & - & - & _- & _- & - & - & _- & $0.1723^{b}$ & - \\
\hline
\end{tabular}

Following the formalism in Eqs. (1)-(10), we calculated physical parameters (see Table 3 ) for the observed galaxy sample, using the estimated redshifts e.g., radial velocities $v_{\text {rad }}$, proper distances $D$, radii $r$ in physical units (proper length), scale-length $h$ in physical units (proper length), absolute $B$-band magnitudes $M_{B}$ and luminosities $L_{B}$. Equations (2) and (4) were used for galaxies having distances $z \geq 0.1$ :

$$
\begin{aligned}
v_{\text {rad }}[z<0.1] & =c z \\
v_{\text {rad }}[z \geq 0.1] & =c \frac{(1+z)^{2}-1}{(1+z)^{2}+1} \\
D[z<0.1] & =\frac{v_{\text {rad }}}{H_{0}} \\
D[z \geq 0.1] & =\frac{2 c}{H_{0}} \frac{1}{1+z}\left[(1+z)-(1+z)^{1 / 2}\right] .
\end{aligned}
$$

For the calculations we assumed a Hubble constant $H_{0}=$ $71 \mathrm{kms}^{-1} \mathrm{Mpc}^{-3}$ (Spergel et al. 2003):

$$
\begin{aligned}
& r[z<0.1]=2 D \tan \frac{r_{\text {arcsec }}}{2} \\
& h[z<0.1]=2 D \tan \frac{\alpha_{B_{\mathrm{W}}}}{2}
\end{aligned}
$$

$$
\begin{aligned}
& r[z \geq 0.1]=d=\frac{r[\mathrm{rad}] D}{1+z} \\
& h[z \geq 0.1]=d=\frac{\alpha_{B_{\mathrm{W}}}[\mathrm{rad}] D}{1+z} .
\end{aligned}
$$

The radius $r$ (proper length $d$ ) in physical units as well as the scale-length $h$ (proper length) in physical units of galaxies with redshifts above $z>0.1$ were calculated using Eqs. (7) and (8) (Longair 1998). The absolute magnitudes result from the distance modulus (see Eqs. (9); Driver et al. 1994) using the proper distance $D$ (see Eqs. (3) and (4)). We used the absolute magnitudes to estimate the $B$-band luminosities $L_{B}$ in units of solar luminosities (see Eq. (10)):

$M_{v}=m_{v}-5 \log _{10}\left[\frac{2 c}{H_{0}}\left((1+z)-(1+z)^{1 / 2}\right)\right]-25-2.5 z$

$M_{B}=4.87-2.5 \log \left(\frac{L}{L_{\odot}}\right)$.

We derived the physical parameters for the whole HDF-S galaxy sample, including LSB J22324-60520 for which we detected no emission lines. This galaxy shows a spectrum of an elliptical 
Table 3. Measured (e.g., $r_{\mathrm{m}}$ measured radii) and calculated parameters of the observed HDF-S galaxy sample. The parameters in Cols. 4, 5, 7, 8, 9 and 10 are calculated using Eqs. (1) to (10) (see also description in Sect. 3.1).

\begin{tabular}{|c|c|c|c|c|c|c|c|c|c|}
\hline "NAME & $\begin{array}{l}\text { RA } \\
\text { [hh:mm:ss.s] }\end{array}$ & $\begin{array}{l}\text { Dec } \\
\text { [dd:mm:ss] }\end{array}$ & $\begin{array}{l}v_{\mathrm{rad}} \\
{\left[\mathrm{km} \mathrm{s}^{-1}\right]}\end{array}$ & $\begin{array}{l}d \\
{[\mathrm{Mpc}]}\end{array}$ & $\begin{array}{l}r_{\mathrm{m}} \\
{[\operatorname{arcsec}]}\end{array}$ & $\begin{array}{l} \\
{[\mathrm{kpc}]}\end{array}$ & $\begin{array}{l}h \\
{[\mathrm{kpc}]}\end{array}$ & $\begin{array}{l}M_{B} \\
{[\mathrm{mag}]}\end{array}$ & $\begin{array}{l}L_{B} \\
{\left[10^{9} L_{\odot}\right]}\end{array}$ \\
\hline LSB J22311-60503 & $22: 31: 13.0$ & $-60: 50: 34$ & $33854 \pm 78$ & $503.7 \pm 25.1$ & 12.9 & $31.5 \pm 1.6$ & $6.2 \pm 0.3$ & $-20.36 \pm 0.07$ & 12.36 \\
\hline LSB J22324-60520 & $22: 32: 41.8$ & $-60: 52: 07$ & 46717 & 638.0 & 8.5 & 7.15 & 1.25 & -20.17 & 10.38 \\
\hline LSB J22325-60155 & $22: 32: 52.2$ & $-60: 15: 58$ & $15780 \pm 69$ & $221.2 \pm 11.0$ & 17.2 & $18.4 \pm 0.9$ & $4.6 \pm 0.2$ & $-16.90 \pm 0.09$ & 0.51 \\
\hline LSB J22 & $22: 3$ & -60 & & $235.0 \pm 11.7$ & 15.1 & $17.1 \pm 0.9$ & $2.5 \pm 0.1$ & $31 \pm 0.08$ & 1.87 \\
\hline LSB J2 & $22: 3$ & $-60: 2$ & 159 & $196.4 \pm 10.0$ & 13.8 & 0.7 & $4.3 \pm 0.1$ & -17 & 0.83 \\
\hline LSB J & & -60 & & 50 & 10.8 & 1.2 & 0.3 & $-1 \xi$ & 2.61 \\
\hline & 22 : & -60 : & & & 10.8 & & 7.3 & $-1^{\prime}$ & 1.14 \\
\hline & $22: 3$ & $-60:$ & & 679. & 7.6 & 1.1 & $6.2 \pm$ & $2 \pm 0.13$ & 7.52 \\
\hline LSB J22355-60183 & $22: 35: 58.3$ & $-60: 18: 40$ & $35149 \pm 57$ & $525.2 \pm 26.1$ & 7.8 & $17.7 \pm 0.9$ & $5.3 \pm 0.3$ & $-19.15 \pm 0.13$ & 4.06 \\
\hline
\end{tabular}
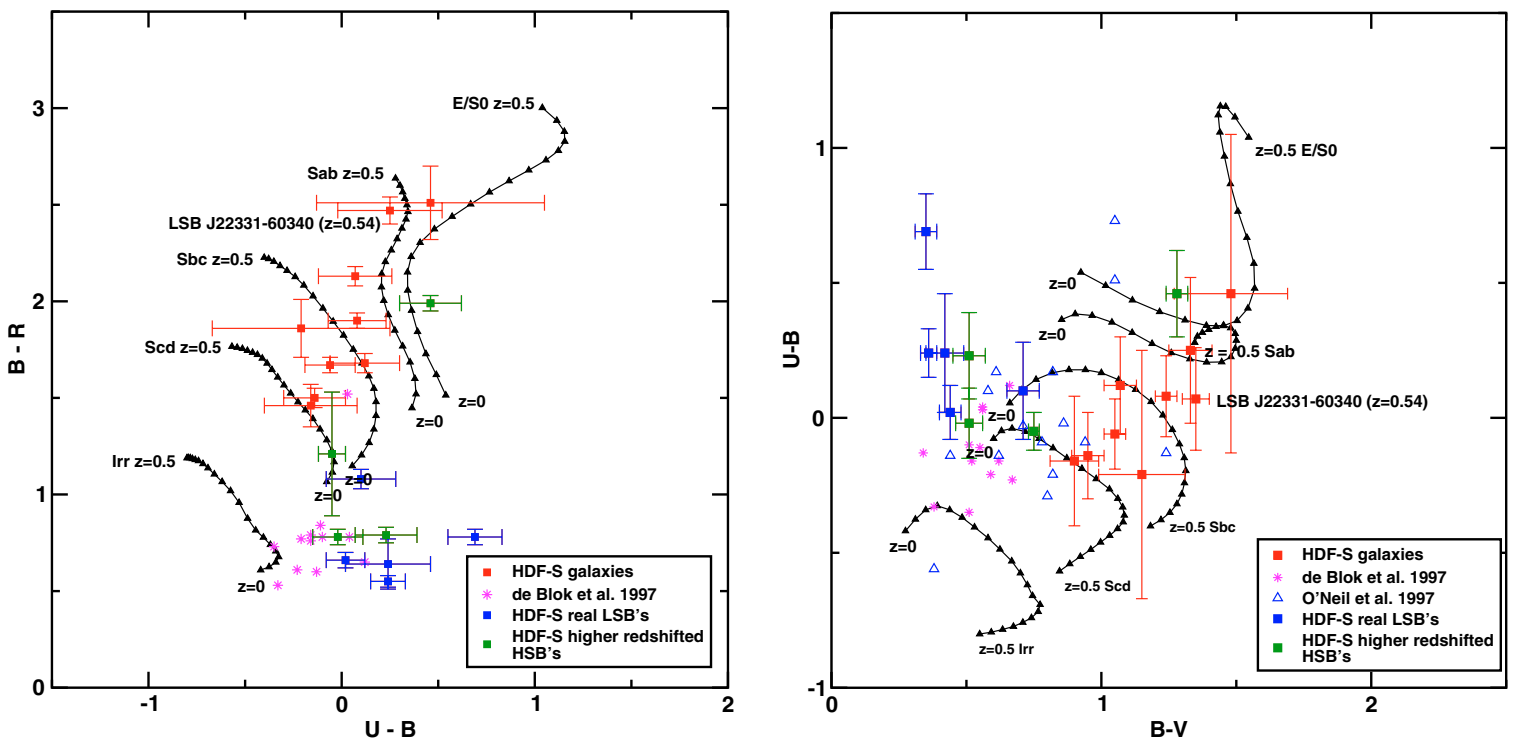

Fig. 2. Color-color diagrams $U-B$ vs. $B-R$ and $B-V$ vs. $U-B$ accounting for the spectroscopically derived distance information. From the selected LSB candidates (blue + green squares), $56 \%$ of the galaxies are found to be true LSBs (blue squares) and $44 \%$ are higher redshifted HSB galaxies (green squares). Two of the higher redshifted HSBs are located at $z \sim 0.1$. They follow the redshift tracks of the "normal" HSB galaxies and therefore should not be selected as LSB candidates.

galaxy with no ongoing star formation. Therefore, we estimated the redshift by measuring the wavelength of the CaII-K absorption line and comparing this value to the rest frame wavelength $\left(\lambda_{0}=3933 \AA\right)$. In the next sections we will discus the properties of the spectroscopically verified LSB galaxies found in the HDF-S.

\subsection{The HDF-S LSB galaxies in the color-color space}

In order to preselect LSB galaxies for spectroscopic follow-up observations efficient methods are required to distinguish candidates from, e.g., objects with low surface brightness caused by cosmological dimming. In a previous paper we have described such a method using a color-color selection criterion (Haberzettl et al. 2007). This selection criterion is based on the different location of the LSB candidates in comparison to the HSB redshift tracks in the color-color space (see also Fig. 2). With the spectroscopic distance information we have now at hand for our LSB candidate sample we will discuss the efficiency of this method.

Using this color-color selection we were able to clean our final sample from higher redshifted background sources (red squares in Fig. 2) which mimic LSB galaxies due to cosmological dimming effects, the so called "Tolman-Dimming" (Hubble \& Tolman 1935):

$$
\mu_{v_{0}}=\frac{\mu_{v_{1}}}{2.5 \log (1+z)^{4}} \text {. }
$$

For the selection process we used two different color-color diagrams $(B-V$ vs. $U-B$ and $U-B$ vs. $B-R)$ in combination with redshift tracks adopted from a work of Liu \& Green (1998).

After removing the higher redshifted background HSB galaxies from the candidate sample we were able to show, that most of the selected LSB galaxy candidates (7 galaxies, blue and green squares) have a distinct different location in the color-color diagrams compared to the redshift tracks of the five standard Hubble types (see Fig. 2).

The LSB candidates have a redder $U-B$ color $(U-$ $B \geq-0.05 \mathrm{mag}$ ), whereas the $B-R$ and $B-V$ colors are shifted into the blue color range ( $B-R \leq 1.08 \mathrm{mag}, B-V \leq 0.71 \mathrm{mag})$.

Using the spectroscopically obtained distances we are now able to finally select the real LSB galaxies from our HDF-S LSB candidate sample (see Table 4). It turned out that $\sim 45 \%$ of the preselected LSB candidates (4 out of 9) have redshifts $z \leq 0.1$ (blue squares in Fig. 2). These galaxies are local 
Table 4. List of the galaxies which were selected as LSB candidates using color-color diagrams. The last column marks whether the candidate, after analyzing the spectroscopic information, is a true LSB galaxy or not. The central surface brightness in Col. 6 is corrected against fading due to "Tolman-Dimming".

\begin{tabular}{|c|c|c|c|c|c|c|}
\hline Name & $\begin{array}{l}U-B \\
{[\mathrm{mag}]}\end{array}$ & $\begin{array}{l}B-V \\
{[\mathrm{mag}]}\end{array}$ & $\begin{array}{l}B-R \\
{[\mathrm{mag}]}\end{array}$ & $\begin{array}{c}\mu_{0} \\
B_{\mathrm{W}} \operatorname{mag} \operatorname{arcsec}^{-2}\end{array}$ & $\begin{array}{c}\mu_{0}(\text { corr }) \\
B_{\mathrm{W}} \mathrm{mag}^{2} \operatorname{arcsec}^{-2}\end{array}$ & LSB galaxy \\
\hline LSB J22311-60503 & -0.05 & 0.75 & 1.21 & 22.4 & 21.9 & no \\
\hline LSB J22324-60520 & 0.46 & 1.28 & 1.99 & 22.3 & 21.6 & no \\
\hline LSB J22325-60155 & 0.02 & 0.44 & 0.66 & 23.3 & 23.3 & yes \\
\hline LSB J22330-60543 & 0.24 & 0.36 & 0.55 & 22.2 & 22.2 & yes \\
\hline LSB J22343-60222 & 0.24 & 0.42 & 0.64 & 23.3 & 23.3 & yes \\
\hline LSB J22352-60420 & 0.10 & 0.71 & 1.08 & 23.2 & 22.7 & yes \\
\hline LSB J22353-60311 & 0.69 & 0.35 & 0.77 & 23.4 & 23.4 & yes \\
\hline LSB J22354-60122 & -0.02 & 0.51 & 0.78 & 22.5 & 21.8 & no \\
\hline LSB J22355-60183 & 0.23 & 0.51 & 0.79 & 22.5 & 22.0 & no \\
\hline
\end{tabular}

galaxies. No surface brightness correction against cosmological dimming has to be applied and, therefore, they are real LSB galaxies. One of the candidates (LSB J22352-60420) has a slightly larger redshift of $z=0.12$. However, after correcting the central surface brightness against "Tolman-Dimming", this galaxy still remains as a LSB galaxy, showing a central surface brightness below $\mu_{0}>22.5 B \mathrm{mag} \operatorname{arcsec}^{-2}$ (see Table 4). Finally the majority ( 5 out of 9 ) of the preselected LSB candidates turned out to be genuine LSB galaxies. For our final LSB galaxy sample we include all galaxies having central surface brightnesses $\mu_{0} \geq 22.2 \mathrm{mag} \mathrm{arcsec}^{-2}$, which is more than $2 \sigma$ below the Freeman value of $\mu_{0}=21.65 \pm$ $0.3 \mathrm{mag} \mathrm{arcsec}^{-2}$ (Freeman 1970). The other four selected candidates (green squares) show redshifts $z \geq 0.1$. After correcting against the "Tolman-Dimming" effect, the central surface brightnesses of these candidates came out to have values $\mu_{0} \leq$ $22.2 \mathrm{mag} \operatorname{arcsec}^{-2}$. Therefore, these galaxies have to be considered as higher redshifted "normal" HSB galaxies.

After a first inspection, it turned out that by using the color-color selection criterion, only $56 \%$ of the selected galaxies are genuine LSB galaxies. However, this selection criterion also resulted in nearly the same amount (44\%) of higher redshifted "normal" HSB background galaxies. Therefore, this would not be a very reliable method in order to preselect a LSB subsample from a large sample of galaxies. A more carefully analysis of the selection in Fig. 2 shows that two of the higher redshifted HSB galaxies (LSB J22311-60503, LSB J22324-60520, green squares) which were also selected as LSB candidates do follow the redshift tracks. After correcting the surface brightnesses against "Tolman-Dimming", these galaxies appear to be higher redshifted "normal" HSB galaxies. Their classification as LSB candidates was too optimistic, due to the relatively large uncertainties in the photometric redshift determination. Two additional galaxies (LSB J22354-60122, LSB J22355-60183, green squares) which are clearly separated from the redshift tracks also turned out to be located at redshifts $z \geq 0.1$. After correcting their central surface brightnesses against "Tolman-Dimming", these galaxies also came out to be HSB galaxies. However, these two galaxies do not behave like "normal" HSBs. They must have extreme colors in order to be moved to this location in the color-color space.

Excluding now the two higher redshifted galaxies, located along the redshift tracks, the method yields 5 LSB galaxies $(71 \%)$ out of a sample of 7 selected galaxies. Only 2 out of 7 galaxies (29\%) are higher redshifted HSB galaxies. This relatively high success rate makes the described method a reasonable tool to select LSB galaxies from larger samples of galaxies.

\subsection{Volume densities of the HDF-S LSB galaxies}

One result of the search for LSB galaxies in the HDF-S is a number surface density of $8.5 \mathrm{LSB}$ galaxies per $\mathrm{deg}^{2}$. This is more than two times higher than found in previous surveys (e.g., O'Neil et al. 1997a,b; Dalcanton et al. 1997, which give 4 LSB galaxies $\mathrm{deg}^{-2}$ ). This number density is not very meaningful, since due to the use of much more sensitive data $\left(\mu_{\lim } \approx\right.$ 29 mag $\operatorname{arcsec}^{-2}$ ), a much larger search volume was covered.

In order to also include the covered volume $V$, it is more convenient to calculate the volume density $n$ for the derived sample. This we have done following a method from McGaugh (1996). With this method, surface brightness corrected and normalized volume densities $\Phi$ could be estimated. At this point we like to mention that we are aware that the method described in the following is a statistical method to compare the surface brightness distribution of different galaxy samples. Our sample of five LSB galaxies plus three extreme LSB candidates (description see below) is not a statistically significant galaxy sample. Thus, any interpretations drawn from this analysis have to be discussed very carefully. However, using this kind of analysis give hints about how our results fit into the existing overall picture of LSB galaxies.

The relative volume density of LSB galaxies $n_{\mathrm{LSB}}$ in relation to the volume density of a well known sample of HSB galaxies $n_{\mathrm{HSB}}$ is described as:

$$
\begin{aligned}
& \frac{n_{\mathrm{LSB}}}{n_{\mathrm{HSB}}}=\frac{N_{\mathrm{LSB}}}{N_{\mathrm{HSB}}}\left(\frac{(V / \Omega)_{\mathrm{HSB}}}{(V / \Omega)_{\mathrm{LSB}}}\right) \\
& \Phi_{\mathrm{norm}}=\frac{n_{\mathrm{LSB}}}{n_{\mathrm{HSB}}}=\frac{N_{\mathrm{L}}}{N_{\mathrm{H}}}\left(\frac{\theta_{1}^{\mathrm{L}}}{\theta_{1}^{\mathrm{H}}}\right)^{3}\left(\frac{h_{\mathrm{H}}}{h_{\mathrm{L}}}\right)^{3} \frac{\left(\mu_{1}^{\mathrm{H}}-\mu_{0}\right)^{3}}{\left(\mu_{1}^{\mathrm{L}}-\mu_{0}^{\mathrm{L}}\right)^{3}} .
\end{aligned}
$$

Most of the parameters in Eq. (13) can be derived without having distance information for the sample galaxies. The only exception is the scale-length $h$ in physical units. For the calculation of the volume density, the assumption is made that the scale-length $h_{\mathrm{L}}$ of the derived LSB galaxy sample is comparable to the scalelength $h_{\mathrm{H}}$ of the sample of HSB galaxies to which the sample will be normalized $\left(h_{\mathrm{H}} / h_{\mathrm{L}}=1\right)$. If, on average, HSB galaxies had a larger scale-length, the value of $n_{\mathrm{LSB}} / n_{\mathrm{HSB}}$ would increase. Using this assumption, we were able to calculate lower limits for the normalized volume densities of our LSB galaxy sample.

For our HDF-S LSB sample, we determined a number surface density of $N_{\mathrm{L}}=8.5 \mathrm{deg}^{-2}$ as well as a surface brightness limit of $\mu_{1}=29.0 \mathrm{mag} \operatorname{arcsec}^{-2}$. We chose a diameter limit of $\theta_{1} \geq 10.8$ arcsec. Due to the small number of objects, we used a central surface brightness bin size of $1 \mathrm{mag} \operatorname{arcsec}^{-2}$ in order to estimate normalized volume densities. For the first two surface 
brightness bins (22.5 mag $\operatorname{arcsec}^{-2}$ and $\left.23.5 \mathrm{mag} \mathrm{arcsec}^{-2}\right)$ we calculated the volume densities using a field size of $0.59 \mathrm{deg}^{2}$. Therefore, we accounted for the fact that all of these galaxies were found in the smaller field covered by the Goddard data (see Haberzettl et al. 2007, for more details). For the extreme LSB candidates (last two surface brightness bins) we had to choose the larger NOAO field of $0.76 \mathrm{deg}^{2}$. Finally we normalized the sample with respect to the well studied galaxy sample of de Jong (1996). This is a statistically complete sample of 86 disk galaxies. The sample was selected from the UGC catalog including galaxies with diameters $\theta$ larger than 2 arcmin and a minor to major axis ratio larger than 0.625 . The survey covered an area of $1.57 \mathrm{srad} \equiv 5154.3 \mathrm{deg}^{-2}$. The surface brightness limit of the catalog is about $\mu_{1}=26.5 \mathrm{~B} \mathrm{mag} \operatorname{arcsec}^{-2}$. We calculated the errors for the surface brightness corrected volume densities using Poisson statistics and Gaussian errors perturbation:

$$
\begin{aligned}
\sigma_{\mathrm{L}} & =\sqrt{\frac{1}{\mathcal{N}_{\mathcal{L}}}} \\
\Delta \Phi_{\text {norm }} & = \pm \sqrt{\left(\frac{\partial \Phi_{\text {norm }}}{\partial N_{\mathrm{L}}} \sigma_{\mathrm{L}}\right)^{2}+\left(\frac{\partial \Phi}{\partial \mu_{0}^{\mathrm{H}}} \Delta \mu_{0}^{\mathrm{H}}\right)^{2}} .
\end{aligned}
$$

For comparison we also plotted the surface brightness corrected volume densities (estimated following the same method) for two additional, well known LSB samples of O'Neil et al. (1997a) (Texas Survey) and Impey et al. (1996). The Texas survey covered an area of $27 \mathrm{deg}^{2}$ on the sky. In this area, a sample of 127 LSB galaxies with diameters larger than 13.5 arcsec was derived. The survey reached a surface brightness limit of $\mu_{1}=27 \mathrm{~B} \mathrm{mag} \operatorname{arcsec}^{-2}$. The search area of the survey of Impey et al. (1996) covers about $768 \mathrm{deg}^{2}$ of the sky. The field is located around the equatorial strip over a declination range of $\pm 2^{\circ}$. The survey reached a surface brightness limit of $\mu_{1}=26 \mathrm{~B} \mathrm{mag} \operatorname{arcsec}^{-2}$. The derived sample consists of 693 galaxies larger than 30 arcsec. For both surveys we used a surface brightness bin size of $0.5 \mathrm{mag} \operatorname{arcsec}^{-2}$.

The results of the discussed estimations of the normalized, surface brightness corrected volume densities is plotted in Fig. 3 . In this diagram, we were able to show that the central surface brightness distribution for our HDF-S LSB galaxy sample (LSB J's, blue triangles) follows the flat distribution of the other LSB surveys. At lower central surface brightnesses (more than $3 \sigma$ below the Freeman value of $\mu_{0}=21.65 \pm 0.35 \mathrm{mag} \mathrm{arcsec}^{-2}$ ), the number of LSB galaxies is much higher than expected from the Freeman Law (purple line). The volume density of the HDF-S LSB galaxy sample derived for the surface brightness bin $\mu=22.5 \mathrm{mag} \operatorname{arcsec}^{-2}$ is slightly lower compared to the values of O'Neil et al. (1997a), Impey et al. (1996) and de Jong (1996). This is maybe the result of incompleteness at the upper surface brightness limit of the HDF-S galaxy sample (upper selection limit $\mu(0)=22.0 \mathrm{mag} \mathrm{arcsec}^{-2}$ ) and/or due to very low number statistics for the HDF-S LSB sample. The Texas survey also shows an incompleteness around the Freeman value $\left(\mu=21.5 \mathrm{mag} \operatorname{arcsec}^{-2}\right)$. The large error bar of our survey at $\mu=25.5$ mag $\operatorname{arcsec}^{-2}$ resulted from low number statistic for this bin (1 LSB galaxy). However, the one object at this value and the two objects at the next bin $\left(\mu=26.5 \mathrm{mag} \mathrm{arcsec}^{-2}\right)$ still have a big implication for the volume density, due to the low volume over which such extreme Low Surface Brightness galaxies could be detected. To find one of these extreme LSB galaxies in a small field, as covered by the NOAO data $\left(\sim 0.76 \mathrm{deg}^{2}\right)$, has a very low probability. The detection of these objects indicate that the formation of such extreme LSB galaxies is as common

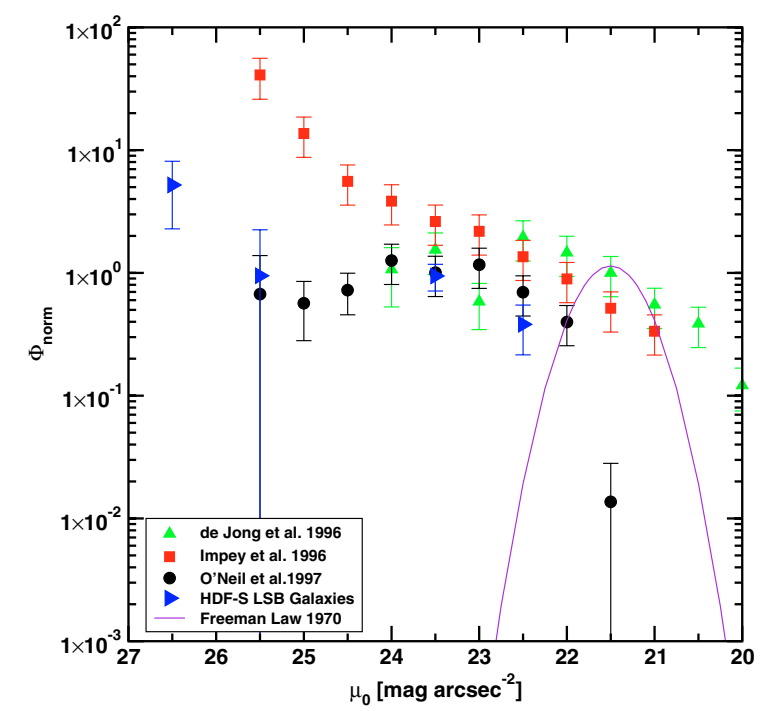

Fig. 3. Surface brightness corrected volume densities for several samples of LSB galaxies and the HSB galaxy sample of de Jong (1996, green triangle). The LSB galaxy sample derived in the HDF-S is represented by the blue triangles. For a comparison, the distribution of the galaxies expected from the Freeman Law (Freeman 1970, purple line) is plotted. The diagram shows that in contrast to the expected distribution of the Freeman Law, the distribution for the LSB galaxies stays flat down to very low central surface brightnesses.

in the Universe as the formation of the higher surface brightness objects found in the surveys.

\subsection{Metallicities}

Since we were not able to detect emission lines sensitive for direct abundance measures (e.g. O[III] 24363 ), we have to use a different approach to get information about the metallicities in our LSB galaxy sample. One commonly used method to derive abundance information is the strong-line method of Pagel et al. (1979). We used this so called R23-method in combination with the semi-empirical calibration of McGaugh $(1991,1994)$ to get an estimation of the oxygen abundances in the derived HDF-S LSB galaxies. With the R23-method we were able to estimate metallicities just using measurements of the most prominent emission lines $\mathrm{H} \beta$, [OII], and the [OIII]-doublet (see Table 5).

$$
\begin{aligned}
& R_{23}=\frac{[\mathrm{OII}] \lambda 3727+[\mathrm{OIII}] \lambda \lambda 4959,5007}{\mathrm{H} \beta} \\
& O_{32}=\frac{[\mathrm{OIII}] \lambda \lambda 4959,5007}{[\mathrm{OII}] \lambda 3727} .
\end{aligned}
$$

The semi-empirical calibration which we used for this abundance estimation is described in more detail in Appendix A of de Naray et al. (2004). To discriminate between the upper and lower branch of this solution (see Fig. 4) we used the [NII] over [OII] relation as described in McGaugh (1994). Objects with $\log ([\mathrm{NII}] /[\mathrm{OII}])>-1$ had abundances derived from the upper brunch, while for $\log ([\mathrm{NII}] /[\mathrm{OII}])<-1$ we used the solution from the lower branch.

Despite of the large uncertainties for the derived emission line intensities we derived subsolar oxygen abundances for all LSB galaxies in our sample (see Table 5). The oxygen abundance range between $\sim 1 / 2 Z_{\odot}$ and $\sim 1 / 10$ th $Z_{\odot}$. These low metallicities are typical for LSB galaxies and in good 
Table 5. Results of the strong-line method, derived using the semi-empirical calibration described in McGaugh (1994) and de Naray et al. (2004). Columns 2 to 6 show the measured equivalent width (including errors) for the LSB galaxy sample). Columns 7 to 9 gives the logarithm of the emission line relations as used in the $R_{23}$ method, Col. 10 gives the oxygen abundances and the last column shows the oxygen abundance in terms of solar abundance. The values of $\log ([\mathrm{NII}] /[\mathrm{OII}])$ are derived using upper limits for the $[\mathrm{NII}]$ measurements. The metallicities are derived from the lower branch values for $\log ([\mathrm{NII}] /[\mathrm{OII}])<-1$ and from the upper branch for $\log ([\mathrm{NII}] /[\mathrm{OII}])>-1$.

\begin{tabular}{|c|c|c|c|c|c|c|c|c|c|c|}
\hline \multirow[t]{2}{*}{ Name } & $\overline{\overline{\mathrm{H}} \beta}$ & $\overline{\overline{[\mathrm{OII}]}}$ & 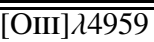 & $\overline{\overline{\text { OIIII] }} \lambda 5007}$ & $\overline{\overline{[\mathrm{NII}]}}$ & $\overline{\log (\text { R23 })}$ & $\overline{\log (\mathrm{O} 32)}$ & $\overline{\overline{[\mathrm{NII}}}$ & $\overline{\overline{l o g}}$ & $\overline{77}$ \\
\hline & [Å] & {$[\AA]$} & {$[\AA]$} & {$[\AA]$} & {$[\AA]$} & & & & & \\
\hline$\overline{\mathrm{LS}}$ & $42 \pm 1.00$ & $11.50 \pm 1.50$ & $5.35 \pm 1.67$ & $12.9 \pm 1.60$ & 0.80 & 71 & 13 & .16 & .30 & 0.13 \\
\hline & $96 \pm$ & & & 19 & 0.25 & & & & & 0.29 \\
\hline & 1.79 & 3.9 & 55 & 53 & 0.26 & 27 & 0.2 & -1.18 & 48 & 0.10 \\
\hline & $67 \pm 0.1$ & & & & 1.11 & & & 4 & & 0.37 \\
\hline LSB J22353-60311 & $1.70 \pm 0.49$ & $5.90 \pm 0.81$ & $1.01 \pm 0.32$ & $2.38 \pm 0.52$ & 0.91 & $0.74 \pm 0.73$ & $-0.24 \pm 0.06$ & -0.81 & $-3.37 \pm 0.74$ & 0.54 \\
\hline
\end{tabular}

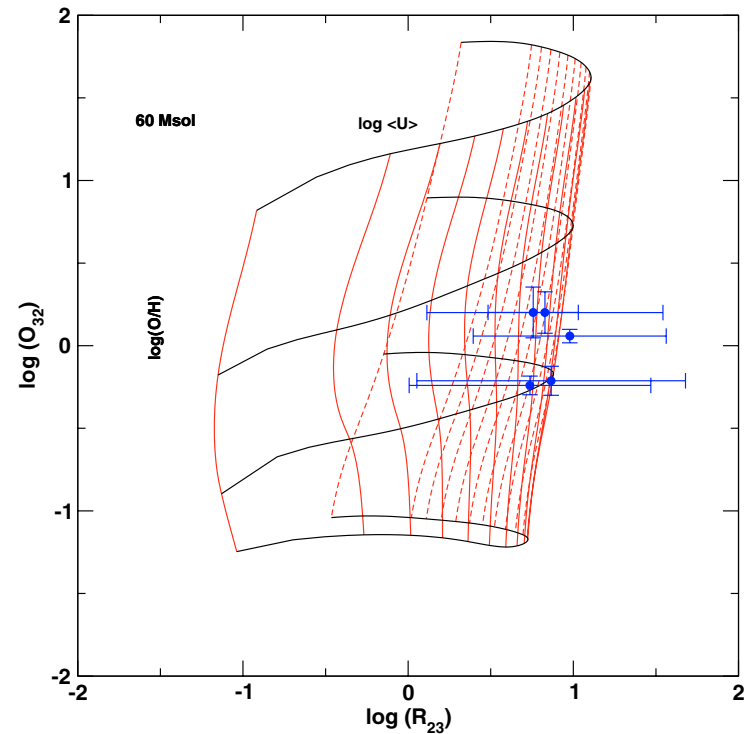

Fig. 4. Metallicities for the five LSB galaxies in the HDF-S, derived using the strong-line method based on the semi-empirical calibration described in McGaugh (1994) and de Naray et al. (2004).

agreement with other measurements (e.g. McGaugh 1994; de Blok \& van der Hulst 1998; de Naray et al. 2004).

\section{Conclusions and summary}

We presented results of spectroscopic follow up observations, obtained using the ESO $3.6 \mathrm{~m}$ telescope, of a sample of 9 LSB galaxy candidates, derived in a $0.59 \mathrm{deg}^{2}$ field around the HDF-S. We used the measured emission lines to determine distances for the observed galaxies in the redshift range of $z \sim 0.05$ to $z \sim 0.16$. The majority of the observed HDF-S sample galaxies is located at redshifts below $z \leq 0.1$, which makes them real LSB galaxies. Using color-color diagrams $(B-V$ vs. $U-B$ and $U-B$ vs. $B-R$ ), we could show that $71 \%$ of the galaxies, having a significant different location compared to the location of the HSB galaxies, are genuine LSB galaxies. This indicates that the use of the location in the color-color diagrams is an efficient method in order to preselect the content of LSB candidates against higher redshifted background galaxies. In the color-color diagrams, the LSB galaxies are shifted to the blue region for the $B-R$ and $B-V$ color and to the red region for the $U-B$ color. This different location in the color-color space seems to be a hint for a different stellar population mix, and therefore, a different Star Formation History (SFH) of the selected LSB galaxies. The shifts could be caused by a stronger
Balmer jump indicating younger stellar population. The shift into the red region of the $U-B$ color could result from a low $\mathrm{UV}$ flux due to a recent low formation rate of $\mathrm{O}, \mathrm{B}$, and A stars. The shift to the blue of the $B-R$ and $B-V$ color also seems to indicate that there exists no strong underlying old stellar population which could cause the shift to the red in the $U-B$ color. To produce this blue-ward shift, a steeper decline of the red part of the stellar continuum emission is needed. Therefore, the red $U-B$ color could also not be the result of a higher metalicity. In this case, the decline in the red part of the continuum emission must be shallower, caused by a stronger underlying old stellar population. The suppression of the $U$-flux due to a large amount of dust could also be excluded. Until now only a few detections of LSB galaxies in the FIR wavelength region at very low levels exist (Hoeppe et al. 1994).

We were also able to estimate oxygen abundances of our five LSB galaxies in the HDF-S, using the strong-line method in combination with the semi-empirical calibration of McGaugh (1991). Despite the large uncertainties, we estimated relatively low oxygen abundances between 0.5 to 0.1 of solar value. This indicates that the sample LSB galaxies are relatively young and unevolved and could explain their location in the color-color space.

The derived LSB sample consists of galaxies with scalelength between $2.5-7.3 \mathrm{kpc}$ and absolute $B$-band magnitudes between $M_{B}=-16.90 \mathrm{mag}$ and $M_{B}=-18.67 \mathrm{mag}$ (see Table 3). From the absolute magnitudes, luminosities were derived in the range of $0.51-2.61 \times 10^{9} L_{\odot}$. Therefore, the sample does not include dwarf galaxies, which is often expected for LSB galaxy samples. All galaxies have distances larger than $z=0.05$. No nearby LSB galaxy could be found, perhaps not surprising given our small survey volume at very low redshift.

In recent years, more sensitive surveys have shown that the Freeman Law (Freeman 1970) was the result of selection effects (see Fig. 3). From these surveys, surface brightness distributions which stay flat down to central surface brightnesses of about $25.5 \mathrm{mag} \operatorname{arcsec}^{-2}$ could be obtained. An estimation of the surface brightness dependent volume density for the HDF-S LSB sample indicate that the results of the HDF-S LSB survey are consistent with results found for other surveys (e.g., O'Neil et al. 1997a,b; Impey et al. 1996; de Jong 1996). Additionally, the distribution could be expanded down to very low surface brightnesses of $\mu_{0}=27 \mathrm{mag} \operatorname{arcsec}^{-2}$. It could be shown that the distribution stays flat also for these very low central surface brightnesses. The results of the HDF-S LSB sample fit very well to the picture drawn by former surveys and lead to the assumption that the LSB galaxies represent a major contribution of the local galaxy population. O'Neil (2000) stated that if the distribution stays flat down to a central surface brightness around $30 \mathrm{mag} \mathrm{arcsec}^{-2}$, up to $50 \%$ of the local galaxy population 
could consist of LSB galaxies. Minchin et al. (2004) showed that $60 \%$ of the population of gas rich disks is represented by the LSB galaxies. All together the LSB galaxies represent a nonneglectable part of the local galaxy population, and therefore, they play an important role for the understanding of the formation and evolution processes of galaxies in general.

Acknowledgements. This research was supported by the DFG Graduiertenkolleg "The Magellanic Systems, Galaxy Interaction and the Evolution of Dwarf Galaxies" (Universities Bonn/Bochum). This work was funded by the DESY/BMBF grant 05 AE2PDA/8. This Paper is based on observations collected at the European Southern Observatory, Chile Prog. Id. 66.A-0154(A). We thank the NOAO Deep Wide-Field Survey team for making the pilot survey data immediately public, and the STIS team at GSFC for the second data set. We also thank Jim Lauroesch for his help with the manuscript and the referee, who helped to improve the paper significantly.

\section{References}

Bell, E. F., Barnaby, D., Bower, R. G., et al. 2000, MNRAS, 312, 470

Dalcanton, J. J., Spergel, D. N., Gunn, J. E., Schmidt, M., \& Schneider, D. P. 1997, AJ, 114, 635

de Blok, W. J. G., \& van der Hulst, J. M. 1998, A\&A, 335, 421

de Blok, W. J. G., van der Hulst, J. M., \& Bothun, G. D. 1995, MNRAS, 274, 235

de Jong, R. S. 1996, A\&AS, 118, 557

de Naray, R. K., McGaugh, S. S., \& de Blok, W. J. G. 2004, MNRAS, 355, 887

Driver, S. P., Phillipps, S., Davies, J. I., Morgan, I., \& Disney, M. J. 1994, MNRAS, 266, 155

Filippenko, A. V. 1982, PASP, 94, 715

Freeman, K. C. 1970, ApJ, 160, 811

Gerritsen, J. P. E., \& de Blok, W. J. G. 1999, A\&A, 342, 655

Haberzettl, L., Bomans, D. J., Dettmar, R.-J., \& Pohlen, M. 2007, A\&A, 465, 95

Hamuy, M., Walker, A. R., Suntzeff, N. B., et al. 1992, PASP, 104, 533

Hoeppe, G., Brinks, E., Klein, U., et al. 1994, AJ, 108, 446
Hubble, E., \& Tolman, R. C. 1935, ApJ, 82, 302

Impey, C. D., Sprayberry, D., Irwin, M. J., \& Bothun, G. D. 1996, ApJS, 105, 209

Kennicutt, R. C. 1989, AJ, 344, 685

Liu, C. T., \& Green, R. F. 1998, AJ, 116, 1074

Longair, M. S. 1998, Galaxy Formation (Berlin, Heidelberg, New York: Springer-Verlag)

Massey, P. 1997, A User's Guide to CCD Reductions with IRAF

Massey, P., Valdes, F., \& Barnes, J. 1992, A User's Guide to Reducing Slit Spectra with IRAF

McGaugh, S. S. 1991, ApJ, 380, 140

McGaugh, S. S. 1993, BAAS, 25, 1384

McGaugh, S. S. 1994, ApJ, 426, 135

McGaugh, S. S. 1996, MNRAS, 280, 337

Minchin, R. F., Disney, M. J., Parker, Q. A., et al. 2004, MNRAS, 355, 1303

Nilson, P. 1973, Uppsala general catalogue of galaxies (Acta Universitatis Upsaliensis. Nova Acta Regiae Societatis Scientiarum Upsaliensis Uppsala Astronomiska Observatoriums Annaler, Uppsala: Astronomiska Observatorium, 1973)

O’Neil, K. 2000 [arXiv:astro-ph/0006253]

O’Neil, K., \& Bothun, G. 2000, ApJ, 529, 811

O’Neil, K., Bothun, G. D., \& Cornell, M. E. 1997a, AJ, 113, 1212

O’Neil, K., Bothun, G. D., Schombert, J., Cornell, M. E., \& Impey, C. D. 1997b, AJ, 114, 2448

Pagel, B. E. J., Edmunds, M. G., Blackwell, D. E., Chun, M. S., \& Smith, G. 1979, MNRAS, 189, 95

Pickering, T. E., Impey, C. D., van Gorkom, J. H., \& Bothun, G. D. 1997, AJ, 114,1858

Roennback, J., \& Bergvall, N. 1995, A\&A, 302, 353

Schlegel, D. J., Finkbeiner, D. P., \& Davis, M. 1998, ApJ, 500, 525

Schombert, J. M., Bothun, G. D., Schneider, S. E., \& McGaugh, S. S. 1992, AJ, 103,1107

Spergel, D. N., Verde, L., Peiris, H. V., et al. 2003, ApJS, 148, 175

van den Hoek, L. B., de Blok, W. J. G., van der Hulst, J. M., \& de Jong, T. 2000, A\&A, 357, 397

van der Hulst, J. M., Skillman, E. D., Smith, T. R., et al. 1993, AJ, 106, 548

van Dokkum, P. G. 2001, PASP, 113, 1420 
L. Haberzettl et al.: Low surface brightness galaxies around the HDF-S. II., Online Material $p 1$

\section{Online Material}


L. Haberzettl et al.: Low surface brightness galaxies around the HDF-S. II., Online Material $p 2$
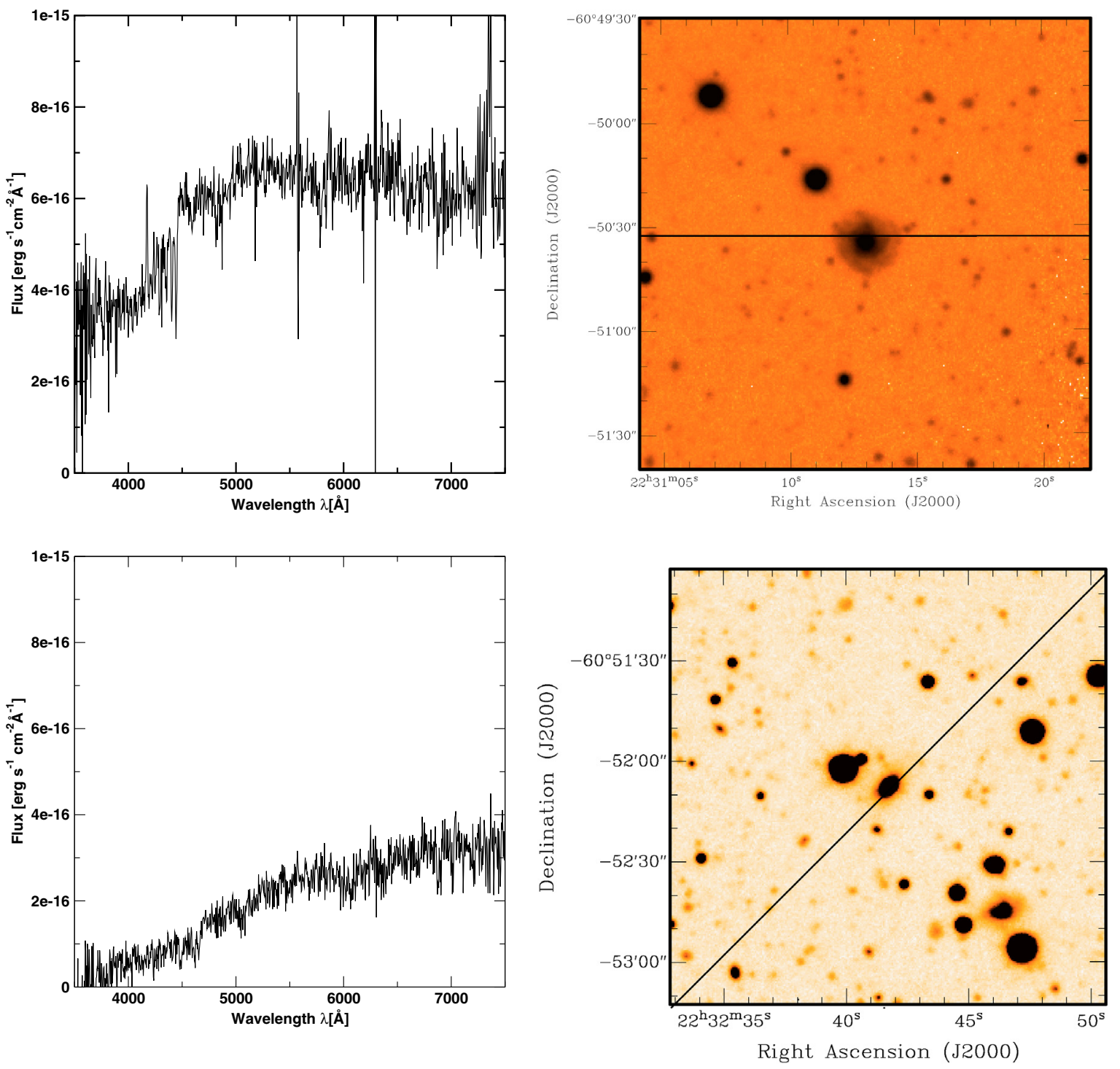

Fig. 1. continued. From top to bottom: LSB J22311-60503 and LSB J22324-60520. 
L. Haberzettl et al.: Low surface brightness galaxies around the HDF-S. II., Online Material $p 3$
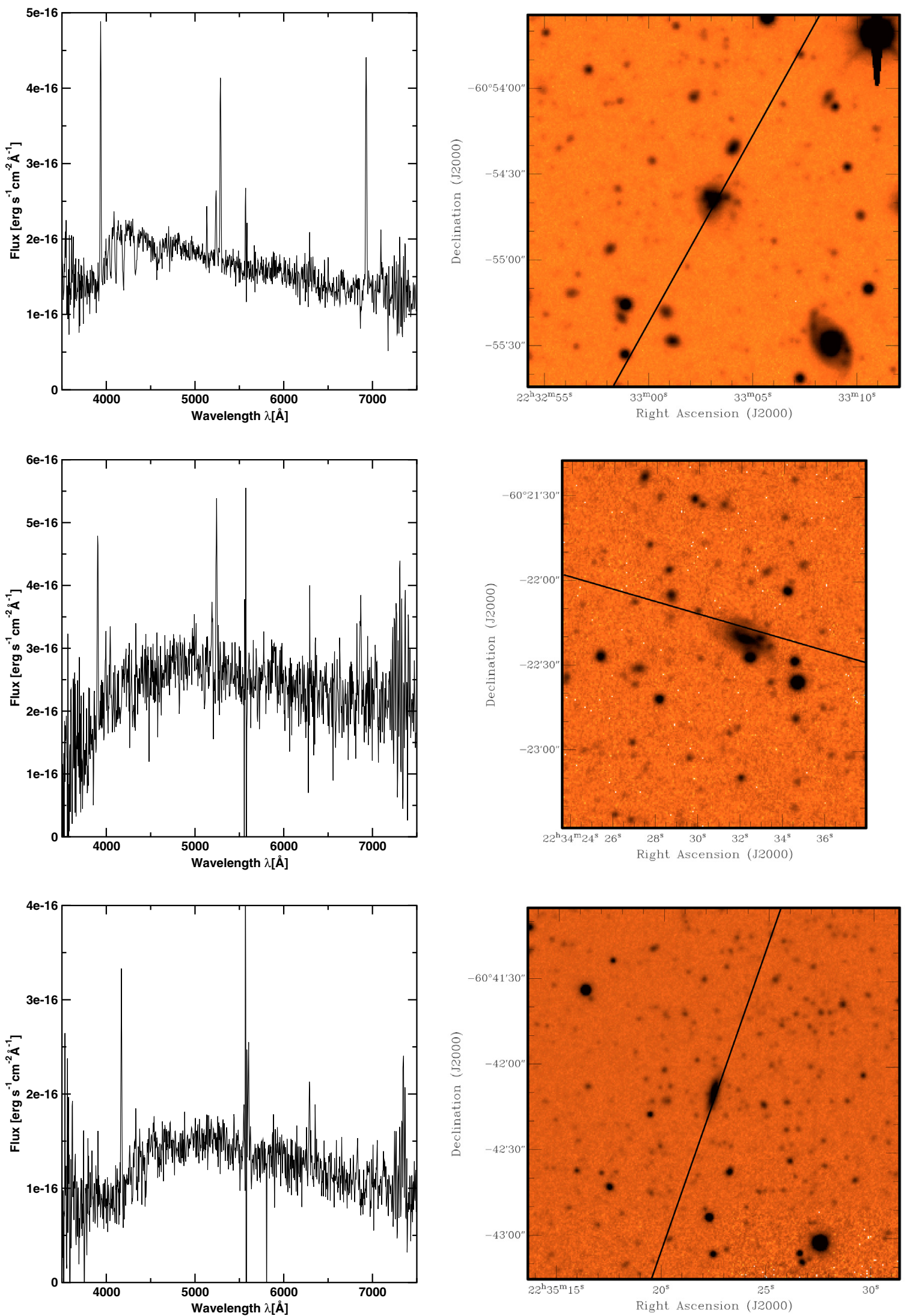

Fig. 1. continued. From top to bottom: LSB J22330-60543, LSB J22343-60222, LSB J22352-60420. 
L. Haberzettl et al.: Low surface brightness galaxies around the HDF-S. II., Online Material $p 4$
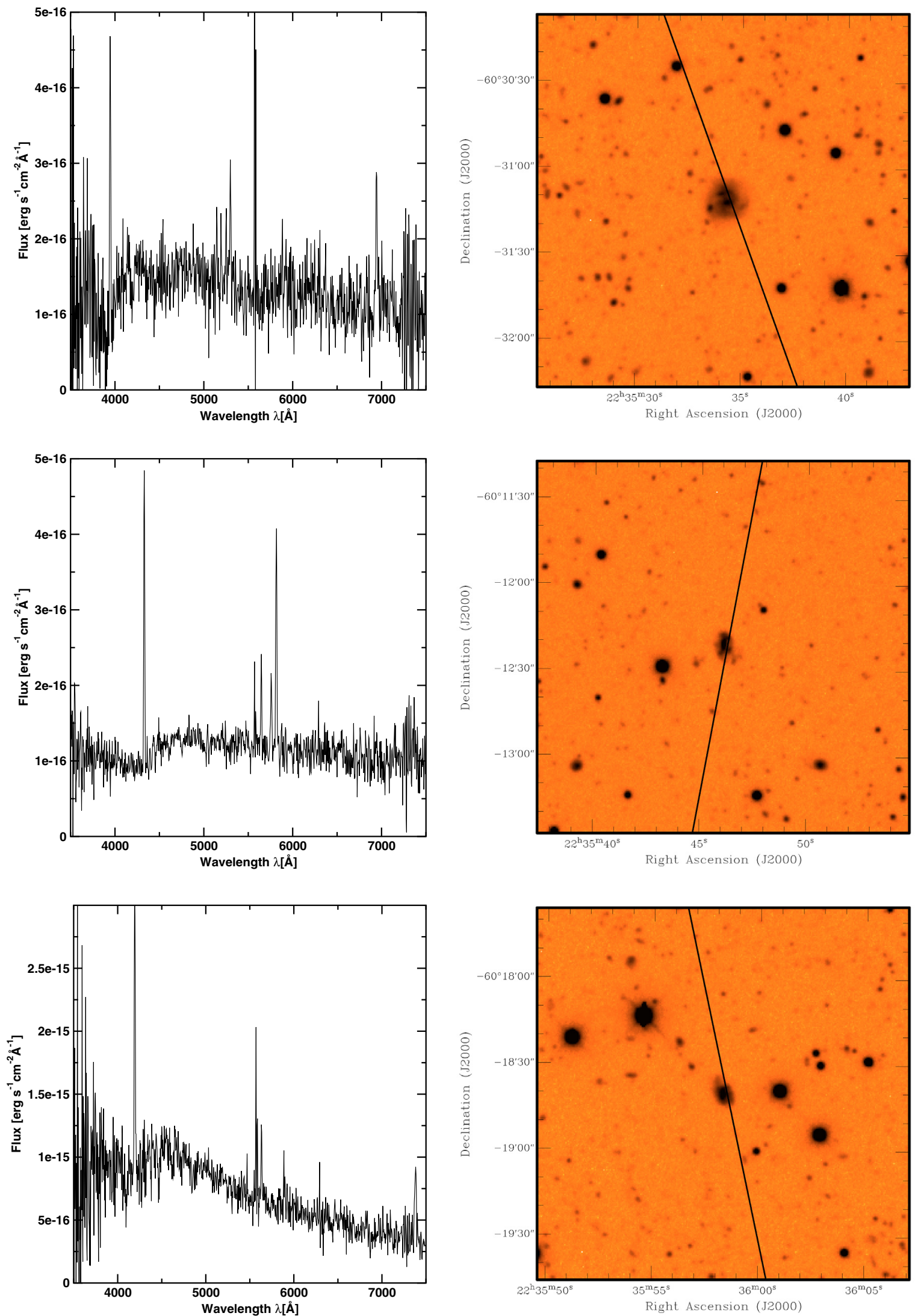

Fig. 1. continued. Form top to bottom: LSB J22353-60311, LSB J22354-60122, LSB J22355-60183. 